\title{
Synthesis and new reaction of sulfur-containing heterocycles for studies of organic conducting materials
}

\author{
Deqing Zhang,* Wei Xu, Chunyang Jia, Hongxiang Li and Daoben Zhu* \\ Organic Solids Laboratory, Center for Molecular Sciences, Institute of Chemistry, Chinese \\ Academy of Sciences, Beijing 100080, China \\ E-mail: dqzhanq@iccas.ac.cn
}

Dedicated to Professor Zhi-Tang Huang on the occasion of his 75th birthday

(received 15 Feb 03; accepted 10 May 03; published on the web 30 May 03)

\begin{abstract}
In this paper, our recent work on the synthesis and new reaction of sulfur-containing heterocycles for studies of organic conducting materials is reviewed. This includes an unusual reaction of zinc complex of 1,3-dithiole-2-thione-4,5-dithiolate [Zn(dmit)2] and its application in the synthesis of new electron donors, and synthetic approaches to tetrathiafulvalene derivatives with hydroxyl and pyridine groups and cross-conjugated TTF-based electron donors as well.
\end{abstract}

Keywords: Tetrathiafulvalene, organic conductors

\section{Introduction}

Since the first synthesis of tetrathiafulvalene (TTF) in 1965,1 and the discovery of metallic conductivity of an organic charge-transfer complex TTF.TCNQ in 1973,2 in particular the finding of the first organic supreconductor in 1979,3 studies of TTF derivatives and their congeneric analogues have received a lot of attention. Various TTF derivatives were designed and synthesized for studies of organic conductors, and even organic superconductors.4 Extensive studies indicate that by increasing the intermolecular interactions and the dimensions of the resulting materials it is possible to effectively stabilize the metallic state at low temperature and hence afford new organic conductors, and even supreconductors. For this reason, TTF derivatives with hydroxyl groups and pyridine groups are interesting because the intermolecular interaction among electron donors and acceptors can be enhanced through the formation of hydrogen bonds with hydroxyl groups or coordination bonds with pyridine groups. Molecular materials with the co-existence of conducting and magnetic properties have also received high attention in recent years.5 One of the approaches to these materials is to exploit the paramagnetic 
metal complexes derived from TTF derivatives with pyridine groups.6 Extended and crossconjugated electron donors are believed to be able to increase the dimensions of the corresponding charge-transfer salts.7 Consequently, TTF-based electron donors with crossconjugated structures become interesting synthetic targets.

One of the important characteristics of TTF derivatives is that they can be converted to the corresponding monocations and dications at easily accessible oxidation potentials. Therefore, TTF derivatives, besides as components of organic conducting materials, are also used to construct donor-acceptor supramolecules for investigations related to molecular electronics, exemplified by the outstanding research work of Stoddart and his coworkers.8 Thus, establishment of efficient and versatile approaches to TTF derivatives is very important. In this paper, we will review our recent work in this respect. This includes: 1) the unusual reaction of zinc complex of 1,3-dithiole-2-thione-4,5-dithiolate [ $\mathrm{Zn}(\mathrm{dmit}) 2$ ] and its application in the synthesis of new electron donors with two TTF units; 2) synthetic approach to TTF derivatives with hydroxyl groups; 3) synthesis of pyridine-substituted TTF molecules; 4) synthesis of TTFbased electron donors with cross-conjugated structures.

\section{Unusual reaction of [ $\mathrm{Zn}(\mathrm{dmit}) 2]$ and its application in the synthesis of new electron donors 9,10}

Direct reaction of [Zn(dmit)2] and suitable electrophilic reagents led to 4,5-bis(alkylthio)- 1,3dithiole-2-thione, an important precursor of TTF-based electron donors. For Scheme 1 see page 200.

However, reaction of [Zn(dmit)2] with 3-picolyl chloride hydrochloride led to a new product after neutralization with K2CO3 and separation with chromatography, besides the expected 4, 5bis(3-picolylthio)-1,3-dithiole-2-thione (1a). Based on the MS, NMR data, the structure of this new compound was established to be 4-(3-picolylthio)-1,3-dithiole-2-thione (2a) (Scheme 1). Furthermore, its structure was confirmed by single crystal X-ray diffraction analysis. If 3-picolyl chloride hydrochloride was first treated by $\mathrm{K} 2 \mathrm{CO} 3$ and then subjected to reaction with [Zn(dmit)2], only 4, 5-bis(3-picolylthio)-1,3-dithiole-2-thione(1a) was yielded (Scheme 1).

The same reactions were conducted with 4-picolyl chloride hydrochloride and [Zn(dmit)2], and similar result was resulted (Scheme 1). Namely, direct reaction of 4-picolyl chloride hydrochloride and [Zn(dmit)2] followed by neutralization led to both $1 \mathrm{~b}$ and 2b. Only compound 1 b was afforded if 4-picolyl chloride hydrochloride was first neutralized by $\mathrm{K} 2 \mathrm{CO} 3$ and then reacted with [Zn(dmit)2]. Since both 3-picolyl chloride hydrochloride and 4-picolyl chloride hydrochloride are weakly acidic, it seems that acidic reaction medium is essential for the formation of $2 \mathrm{a}$ and $2 \mathrm{~b}$. Thus, we assume that compound 3 is the key reaction intermediate for this unusual reaction of [ $\mathrm{Zn}(\mathrm{dmit}) 2]$. The unusual chemical reactivity of 3 was once reported by Rauchfuss et al.11 Accordingly, both 3-picolyl chloride hydrochloride and 4-picolyl chloride hydrochloride may be considered to have two roles for the reaction: weak acid and electrophile. Therefore, by replacing 3-picolyl chloride hydrochloride or 4-picolyl chloride hydrochloride with pyridine hydrochloride and appropriate electrophilic reagent, it would be possible to prepare 
other analogues of 2a and $2 \mathrm{~b}$ with similar approach. Preliminary experimental results were rather encouraging. Namely, pyridine hydrochloride and [Zn(dmit)2] were reacted with electrophilic reagent such as methyl iodide, 3-bromopropionitrile, and benzyl chloride at 50-60oC for 1-2hrs. After separation and purification, the corresponding 4-alkylthio-1,3-dithol-2-thione 2c, 2d and 2e were obtained in addition to the 4,5-bisalkylthio-1,3-dithol-2-thione 1c, $1 \mathrm{~d}$ and $1 \mathrm{e}$ as indicated in Scheme 1 . The reaction yield for 2c and 2e was not high, probably due to the high reactivity of methyl iodide and benzyl chloride which will induce them to react with [Zn(dmit)2] directly forming 1c and 1e respectively. Compound 2d, on the contrary, was separated in fairly high yield. Among the compounds 2a-e, 2d is most interesting since 2-cyanoethyl is a good protecting group widely employed in the chemistry of 1,3-dithiole-2-thione-4,5-dithiolate, 12 and it can be used for the synthesis of other derivatives of 4-alkylthio-1,3-dithole-2-thione. Besides pyridine hydrochloride the hydrochloride salts of triethyl amine, aniline and p-diaminobenzene can also be employed for the reaction to induce the formation of 2a-e.

With pyridine hydrochloride as an example, the following mechanism was tentatively suggested for the unusual reaction of $\mathrm{Zn}(\mathrm{dmit}) 2$ ion (Scheme 2). As mentioned above, compound 3 was supposed to be the key intermediate. Probably with the help of pyridine, homo-cleavage of $\mathrm{S}-\mathrm{H}$ bond generates radical intermediates $3 \mathrm{a}$ and $3 \mathrm{~b}$. For 3a, further cleavage of the neighboring $\mathrm{S}-\mathrm{H}$ bond and intramolecular migration of hydrogen radical lead to radical intermediate 3c and free sulfur. This is in accordance with the separation of S8 in the reaction mixture. Electrontransfer reaction between $3 \mathrm{~b}$ and 3c should afford 4-thio-1,3-dithiole-2-thione anion (3d), which will react with suitable electrophilic reagents to produce the analogues of 2a.

Under similar condition, [Zn(dmit)2] reacted directly with bis-electrophilic reagents such as 1 ,3dibromopropane in the presence of pyridine hydrochloride to afford compounds 4 and 5 with bis(1,3-dithiole-2-thione) groups (see Scheme 3). The reaction yields of 4 and 5 were low (about $20 \%$ ), which was probably due to the formation of compounds with only one 1,3-dithiole-2thione group. Although the yields were not high, the present approach to compounds with bis(1,3-dithiole-2-thione) groups was rather simple in terms of the easiness to obtain the reactants and perform the reaction and separation in comparison with the synthetic method for their analogues.13 These compounds (e.g. 4 and 5) are good precursors for the tetrathiafulavenophanes and other interesting molecules with TTF units.14. For Scheme 2 see page 201.

Since the 2-cyanoethyl group can be easily removed under basic condition,12 $2 \mathrm{~d}$ is a potentially good starting compound for the synthesis of new electron donors and related interesting molecules. For instances, 2d was converted to 6 with $\mathrm{Hg}(\mathrm{OAc}) 2$, and 7 and 8 can be prepared by the conventional cross-coupling procedure effected by tri(isopropyl)

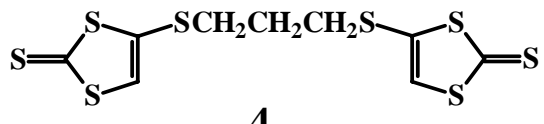

4

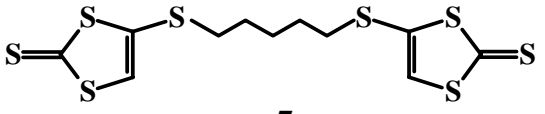

5

\section{Scheme 3}


phosphite in the yields of $73 \%$ and $56 \%$, respectively (Scheme 4). The deprotection of 2 cyanoethyl group resulted in the formation of the corresponding thiolates in situ, which subsequently reacted with bis-electrophilic reagents such as 1,2-dibromoethane to afford new electron donors with two TTF units 9 - 13. Analogues of these new electron donors were synthesized by different multi-step approaches.12 These electron donors are interesting for studies of organic conductors since higher than one dimensional and different stoichiometric charge-transfer complexes may be resulted from them.15 In particular, they may be useful for the construction of molecular spin-ladder systems. ${ }^{16}$ For Scheme 4 see page 202.

\section{Synthetic approach to TTF derivatives with hydroxyl groups 17,18}

The synthesis of new electron donors featuring hydroxymethyl groups 14a-d started from trithione oligomer (see Scheme 5) which was prepared by the oxidation of bis(tetrabutylammonium) bis[2-thioxo-1,3-dithiole-4,5-bis(thiolato)]zincate with iodine. Compounds 15a-b were synthesized by [4+2] cycloaddition between allyl alcohol and 2-butene1,4-diol, respectively, with trithione oligomer, which decomposed to give the unstable 4,5dihydro-1,3-dithiole-2,4,5-trithione monomer on heating. Efforts were also made to synthesize 15a-b by the nucleophilic substitution reaction between 1,3-dithiole-2-thione-4,5-dithiolate (dmit) anion and the corresponding dibromoalkyl alcohols, but no target compounds were detected in the reaction mixture. But, 15a-b could be prepared by the reaction of the corresponding dibromoalkyl alcohols, in which hydroxyl groups were protected, and dmit ion. ${ }^{19}$

\section{For Scheme 5 see page 203.}

The trialkyl phosphite mediated coupling of 1,3-dithiole-2-thione (-ones) is standard chemistry in the construction of TTFderivatives. However, direct coupling of compounds 15a-b failed probably because of the side-reaction between the hydroxy groups and trialkyl phosphite, although triisopropyl phosphite instead of triethyl phosphite was used to hinder such a sidereaction. Thus, 15a-b were converted to 16a-b to protect the hydroxy groups with tertbutyldiphenylchlorosilane. Oxidation of 16a-b with $\mathrm{Hg}(\mathrm{OAc}) 2$ gave $17 \mathrm{a}-\mathrm{b}$ in high yield. The cross coupling reaction between 17a-b and the corresponding 1,3-dithiole-2-thiones afforded compounds 18a-d in moderate yield. Deprotection of 18a-d, using tetrabutylammonium fluoride in $\mathrm{CH} 2 \mathrm{Cl} 2$, afforded target molecules 14a-d in analytically pure form after isolation by column chromatography on silica gel. Since the target molecules $14 a-d$ were easily and quickly oxidized in the crude reaction mixture under air, the deprotection step should be performed under a nitrogen atmosphere and tetrabutylammonium fluoride should not be used in large excess.

\section{Synthesis of pyridine-substituted TTF molecules ${ }^{20,21}$}

As mentioned above, neutralization of 3-picolyl chloride hydrochloride or its analogue and then reaction with [Zn(dmit)2] led to 1,3-dithiole-2-thione derivatives with two pyridine groups. But, it was rather difficult to obtain pure 19 and 20 from the reaction of mercury acetate and these 1,3-dithiole-2-thione compounds with two pyridine groups. This was not fully understood, but it 
was supposed to be due to the coordination of pyridine groups with mercury ion. The direct application of raw products of 19 and 20 made the coupling reaction promoted by trialkyl phosphite more complicated, and the separation of the expected compounds from the mixture was tedious and time-consuming. However, pure 19 and 20 could be easily obtained from compound 21 as showed in Scheme 6. Self-coupling of 19 and 20 in the presence of triisopropyl phosphite afforded 22 and 23 with four pyridine groups in fairly high yields. Cross-coupling of 19 with 4,5-dimethylthio-1,3-dithiole-2-thione and 4,5-ethylenedithio-1,3-dithiole-2-thione respectively led to 24 and 25 with two pyridine units. Similarly, compounds 26 and 27 resulted from 20. Another approach to these electron donors was from compound ${ }^{28.22}$

As mentioned above, oligo(4,5-dihydro-1,3-dithiole-2,4,5-trithione) undergoes thermal depolymerization on heating to give the unstable 4,5-dihydro-1,3-dithiole-2,4,5-trithione monomer, which can act as an effective $4 \pi$ component to form Diels-Alder adducts with dienophiles. Accordingly, the oligomer reacted with 4-vinylpyridine and 2-vinylpyridine leading to the 1,3-dithiole-2-thione compounds 29a and 29b in yields of 15 and 20\% after recrystallization respectively (Scheme 7). Reaction of 29a and 29b with $\mathrm{Hg}(\mathrm{OAc}) 2$ afforded 30a and 30b in high yield. The cross coupling reaction of 4,5-ethylenedithio-1,3-dithiole-2-thione and compounds 30a-b in the presence of triisopropyl phosphite afforded TTF derivatives with one pyridine unit 31a-b. The homo-coupling products of 30a-b were observed in the mass spectra of the reaction mixture, but they were not isolated; they were expected to be a mixture of isomers.

TTF derivatives 32a-b in which the pyridine groups were conjugated with the TTF core were obtained by dehydrogenation of 31a-b with an excess of DDQ in refluxing xylene. Charge transfer complexes were found to be formed during the reaction, which lowered the reaction yields. Similar TTF compounds like 33, in which the pyridine group was connected with the TTF core by the ethylene unit, were prepared by exploiting Wittig type reaction starting from TTF compound with aldehyde unit. ${ }^{23}$ For Scheme 6 and Scheme 7 see pages 204-205.

\section{Synthesis of TTF-based electron donors with cross-conjugated structures ${ }^{24,25}$}

Synthesis of the novel electron donors 34 and 35 with cross-conjugated structures started from the cycloaddition of oligo(1,3-dithiole-2,4,5-trithione) with p-benzoquinone as showed in Scheme 8. This cycloaddition reaction led to the hydroquinone-fused 1,3-dithiole-2-thione 36, not the predicted cyclohexanenedione (36'). This reaction yield was less than 1\% under ordinary reaction condition. But, by using boron trifluoride as catalyst the reaction yield was increased to 5 6\%. Difficulties were also encountered in the purification of 36 because of its poor solubility in most organic solvents. As for the 1,3-dithiole-2-thione containing hydroxylmethyl groups, it was not suitable to use 36 for self-coupling or cross-coupling promoted by trialkyl phosphite. For this reason, 36 was transformed to 37 in which the two phenolic hydroxyl groups were protected by methyl groups. Compound 37 was further transferred to 38 with $\mathrm{Hg}(\mathrm{AcO}) 2$. For this step, only the chloroform was used as the reaction solvents instead of the mixture of chloroform and acetic acid as widely employed, which simplified the work-up stage greatly. As expected, the 
reaction between 38 and triisopropyl phosphite afforded the new electron donor 39a in 67\% yield. Deprotection of methyl groups from 39a with boron tribromide successfully lead to the new electron donor 40a with four phenolic hydroxyl groups in $45 \%$ yield. Similarly, the cross coupling reaction between 38 and substituted 1,3-dithiole-2-thione in the presence of triisopropyl phosphite yield new electron donors 39b-c with unsymmetrical structure, from which new electron donors 40b-c were obtained after removal of methyl groups. As compared to 39a-c, new donors 40a-c with phenolic hydroxyl groups were not so stable, and oxidation to corresponding quinone fused BEDT-TTF derivatives were detected when exposed to atmosphere at room temperature.
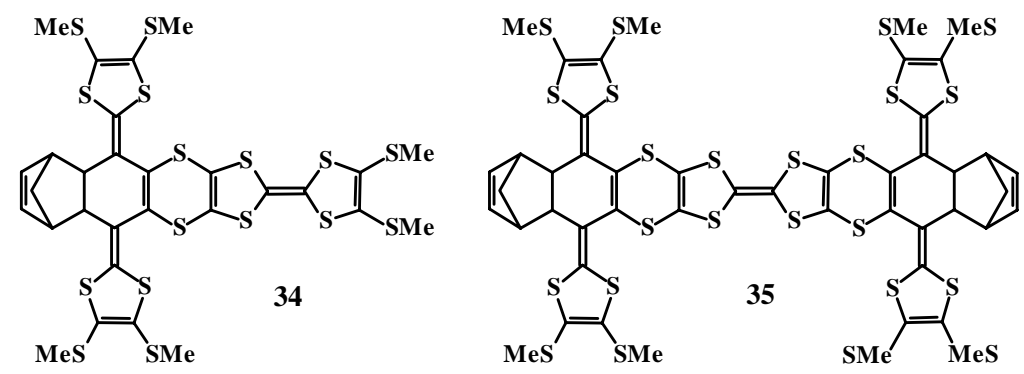

Oxidation of 40b with active $\mathrm{MnO} 2$ yielded 41b. In order to enhance the reactivity of two carbonyl groups, compound 41b was converted to 42 by cycloaddiction with cyclopentadiene. One-pot, two successive Witting-Horner reactions with 42 led to the new electron donor 34 in $45 \%$ yield. Similarly, the new electron donor 35 was obtained by one-pot, four successive Witting-Hornor reactions starting from 41a, which was obtained by oxidation of 40a in 20\% yield (Scheme 8).

Removing the cylcopentadiene moiety by retro-Diels-Alder reaction from 34 would lead to the more appealing donor 34'. The reaction was conducted by heating compound 34 both in solid state and in the solution (o-dichlorobenzene) (Scheme 8) at 160 180oC. Unfortunately, only an unexpected product 43 was detected in the reaction mixture. The chemical structure of 43 was established by mass spectra and NMR data, and further confirmed by single crystal X-ray diffraction analysis. The mechanism of the formation of compound 43 is not very clear at this stage. For Scheme 8 see page 206.

Similar approaches were employed to prepare electron donors with similar extended and cross-conjugated $\pi$-system such as 44 and 45 . However, in these cases, cylcopentadiene moiety can be successfully removed by retro-Diels-Alder reaction.7 For Scheme 8(continued) see page 207.

\section{Summary}

TTF derivatives are important electron donors for studies of organic conducting materials. They are also interesting components of donor-acceptor supramolecules for the research relevant to 
molecular devices. Therefore, synthesis and creation of efficient approaches to TTF derivatives are highly important. Indeed, great progress in this respect has been achieved in recent years.26 In this paper, we summarize our recent work on the synthesis and new reaction of sulfurcontaining heterocycles for studies of organic conducting materials. These results are interesting in terms of both heterocycliv chemistry and materials chemistry. Based on the unusual reaction of [Zn(dmit)2] ion, an efficient and versatile route to 4-alkylthio-1,3-dithilole-2-thione was established. It was also demonstrated that 4-alkylthio-1,3-dithilole-2-thione was a good synthon for the synthesis of TTF-based electron donors. These TTF derivatives featuring pyridine groups will have potential applications in studies of molecular materials showing both conducting and magnetic properties. And electron donors featuring hydroxyl groups, in particular those with extended and cross-conjugated structures are appealing candidates for the preparation of new organic conducting materials.

\section{Acknowledgements}

The present research was financially supported by NSFC (90101025), Chinese Academy of Sciences and State Key Basic Research Program (G2000077505). D.-Q Zhang thanks National Science Fund for Distinguished Young Scholars.

\section{References and Notes}

1. Prinzbach, H.; Berger, H.; Lüttringhaus, A. Angew. Chem. Int. Ed. Engl. 1965, 4, 436.

2. Ferraris, J.; Cowan, D. O.; Walatka, V. J.; Perlstein, H. J. J. Am. Chem. Soc. 1973, 95, 948.

3. Andrieeux, A.; Duromre, C.; Jérome, D.; Bechgaard, K. J. Phys. Lett. 1979, 40, 381.

4. See, for example, Williams, J. M.; Ferraro, J. R.; Thorn, R. J.; Carlson, K. D.; Geiser, U.; Wang, H. H.; Kini, A. M.; Whangbo, M. H. Organic Superconductors (including fullerenes), Prentice Hall, Englewood, Cliffs, NJ.

5. (a) Day, P.; Kurmoo, M.; Mallah, T.; Marsden, I. R.; Friend, R. H.; Pratt, F. L.; Hayes, W.; Chasseau, D.; Gaultier, J.; Bravic, G.; Ducasse, L. J. Am. Chem. Soc. 1992, 114, 10772. (b) Coronado, E.; Galán-Mascarós, J. R.; Gomez-García, C. J.; Laukhin, V. Nature, 2000, 408, 447.

6. Iwahori, F.; Golhen, S.; Ouahab, L. et al. Inorg. Chem. 2001, 40, 6541.

7. (a) Boulle, C.; Desmars, O.; Gautier, N.; Hudhomme, P.; Cariou, M.; Gorgues, A. Chem. Commun. 1998, 2197. (b) Gautier, N.; Mercier, N.; Riou, A.; Gorgues, A.; Hudhomme, P. Tetrahedron Lett., 1999, 40, 5997. (c) Gautier, N.; Gallego-Planas, N.; Mercier, N.; Levillain, E.; Hudhomme, P. Org. Lett. 2002, 4, 961.

8. See, for example, Stoddart, J. F. Acc. Chem. Res. 2001, 34, 410.

9. Jia, C.; Zhang, D.; Xu, W.; Zhu, D. Org. Lett. 2001,1941. 
10. Jia,C.; Zhang, D.; Guo, X.; Wan, S.; Xu, W.; Zhu, D. Synthesis 2002, 15, 2177.

11. (a) Galloway, C. P.; Doxsee, D. D.; Fenske, D.; Rauchfuss, T. B.; Wilson, S. R.; Yang, X. Inorg. Chem. 1994, 33, 4537. (b) Chou, J -H.; Rauchfuss, T. B.; Szczepura, L. F. J. Am. Chem. Soc. 1998, 120, 1805.

12. Svenstrup, N.; Becher, J. Synthesis 1995, 215, and further references therein.

13. (a) Meziere, C.; Fourmigue, M.; Canadell, E.; Clerac, R.; Bechgaard, K.; Auban-Senzier, $P$. Chem. Mater., 2000, 12, 2250. (b) Simonsen, K. B.; Thorup, N.; Becher, J. Synthesis 1997, 1399. (c) Sudmale, I. V.; Tormos, G. V.; Khodorkovsky, V. Y.; Edzina, A. S.; Neilands, O. J.; Cava, M. P. J. Org. Chem. 1993, 58, 1355. (d) Izuoka, A.; Kumai, R.; Sugawara, T. Chem. Lett. 1992, 285.

14. (a) Adam, M.; Enkelmann, V.; Räder, H. J.; Röhrich, J.; Müllen, K. Angew. Chem. Int. Ed. Engl. 1992, 31, 309. (b) Matsuo, K.; Takimiya, K.; Aso, Y.; Otsubo, T.; Ogura, F. Chem. Lett. 1995, 523. (c) Yunoki, S.-R.; Takimiya, K.; Aso, Y.; Otsubo, T. Tetrahedron Lett. 1997, 38, 3017. (d) Takimiya, K.; Imamura, K.; Shibata, Y.; Aso, Y.; Ogura, F.; Otsubo, T. J. Org. Chem. 1997, 62, 5567. (e) Lau, J.; Blanchard, P.; Riou, A.; Jubault, M.; Cava, M. P.; Becher, J. J. Org. Chem. 1997, 62, 4936.

15. (a) Kaplan, M. L.; Haddon, R. C.; Wudl, F. J. Chem. Soc. Chem. Commun. 1977, 388. (b) Adam, M.; Müllen, K. Adv. Mater. 1994, 6, 439. (c) Otsubo, T.; Aso, Y.; Takimiya, K. Adv. Mater. 1996, 8, 203. (d) Nielsen, M. B.; Becher, J. Lieb. Ann. 1997, 2177.

16. Rovia, C. Chem. Eur. J. 2000, 6, 1723, and further references therein.

17. Li, H.; Zhang, D.; Xu, W.; Fan L.; Zhu, D.; Synth. Met. 1999, 106, 111.

18. Li, H.; Zhang, D.; Zhang, B.; Yao, Y.; Xu, W.; Zhu D.; Wang Z. J. Mater. Chem. 2000, 10, 2063.

19. Saygili, N.; Brown, R. J.; Day, P.; Hoelzl, R.; Kathirgamanathan, P.; Mageean, E. R.; Ozturk, T.; Pikington, M.; Qayyum, M. M. B.; Turner, S. S.; Vorwerg, L.; Wallis, J. D. Tetrahedron 2001, 57, 5015.

20. Xu, W.; Zhang, D.; Li, H.; Zhu, D. J. Mater. Chem. 1999, 9, 1245.

21. Jia, C.; Zhang, D.; Xu, W.; Zhu, D. Synth. Met. in press.

22. Liu, S.; Dolder, S.; Pilkington, M.; Decurtins S. J. Org. Chem. 2002, 67, 3160.

23. Andreu, R.; Malfant, I.; Lacroix, P. G.; Cassoux, P. Eur. J. Org. Chem. 2000, 737.

24. Xu, W.; Zhang, D.; Li, H.; Fan L.; Zhu, D. Synthetic Communication 2000, 30, 835.

25. Xu, W.; Zhang, D.; Zhang, B.; Zhu, D.; Fan L.; Jin, X. Synth. Met. 2001, 123, 245.

26. Segura L.; Martín, N. Angew. Chem. Int. Ed. 2001, 40, 1372. 